\title{
UNITARY INVARIANTS FOR COMPACT OPERATORS
}

\author{
BY WILLIAM B. ARVESON ${ }^{1}$
}

\author{
Communicated by M. H. Protter, August 5, 1969
}

We describe in this note how the "boundary representation" technique introduced in [1] leads to a complete classification of compact operators on Hilbert spaces to unitary equivalence (Theorem 3), in terms of a sequence of invariants related to (and generalizing) the numerical range. These invariants are, we feel, vastly simpler than one might have anticipated in so general a situation. Full details will appear in a forthcoming sequel to [1].

1. Boundary representations for spaces of compact operators. Let $L C(\mathfrak{S})$ (resp. $L(\mathfrak{S})$ ) denote the $C^{*}$-algebra of all compact (resp. bounded) operators on a Hilbert space $\mathfrak{S}$, which may be finitedimensional. The following theorem implies, in the terminology of [1], that the identity representation of $L C(\mathfrak{S})$ is a boundary representation for every irreducible linear subspace of $L C(\mathfrak{W}$ ) (we call a set of operators irreducible if it commutes with no nontrivial selfadjoint projections).

THEOREM 1. Let $S$ be an irreducible subset of $L C(\mathfrak{S})$, and let $\phi$ be a completely positive linear map of $L C(\mathfrak{S})$ into $L(\mathfrak{S})$ such that $\|\phi\| \leqq 1$ and $\phi(T)=T$ for every $T$ in $s$. Then $\phi$ is the identity map.

This result is surprising inasmuch as $\delta$ can be a very small subset of $L C(\mathfrak{S})$ a priori. For example, $\delta$ may consist of a single irreducible compact operator. We shall not give the proof of Theorem 1 here, except to say that it is an application of the following.

Lemma. Let $\$$ and $\phi$ satisfy the hypothesis of Theorem 1. Then there is a faithful, completely positive, idempotent linear map $\psi: L(\mathfrak{S}) \rightarrow L(\mathfrak{S})$ such that $\|\psi\| \leqq 1$, and whose compact fixed points coincide with the fixed points of $\phi$.

2. The matrix range of an operator. Let $T$ be a Hilbert space operator, and let $C^{*}(T)$ denote the $C^{*}$-algebra generated by $T$ and the identity. It is well known that, as $\phi$ runs over the state space of $C^{*}(T)$, the complex numbers $\phi(T)$ fill out the closure of the numerical

AMS Subject Classifications. Primary 4665, 4710; Secondary 4745.

Keys Words and Phrases. Operators on Hilbert space, subspaces of $C^{*}$-algebras, spaces of compact operators, boundary representations, matrix-valued numerical range, unitary equivalence.

1 Research supported, in part, by NSF grant GP-5585. 
range of $T$. The following definition generalizes this notion; $M_{n}$ will denote the $C^{*}$-algebra of all complex $n \times n$ matrices, $n=1,2, \cdots$.

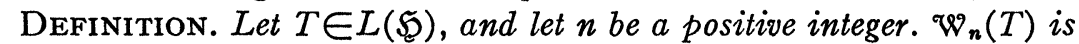
defined as all $n \times n$ matrices of the form $\phi(T)$, where $\phi$ ranges over all completely positive linear maps of $C^{*}(T)$ into $M_{n}$ which preserve the identity.

We remark that it is easy to give an explicit description of $\mathscr{W}_{n}(T)$ in terms of vectors in the underlying space, so that $\mathscr{W}_{n}(T)$ appears as a direct generalization of the ordinary numerical range.

It is significant that $W_{n}(T)$ can be calculated for quite a variety of operators. For example, if $T$ is a normal operator, then it can be shown that $W_{n}(T)$ is the closure of all finite sums $\sum z_{i} K_{i}$, where $z_{i} \in \operatorname{sp}(T)$ (the spectrum of $T$ ) and the $K_{i}$ are positive matrices with sum $I$; i.e., $W_{n}(T)$ is the closed "matrix-valued" convex hull of $\operatorname{sp}(T)$. Still more specifically, if the spectrum of (a unitary operator) $T$ is the entire unit circle, then $W_{n}(T)$ is the closed unit ball in $M_{n}, n=1$, $2, \cdots$ As a second example, let $T$ be an operator on a two-dimensional Hilbert space having a matrix representation

$$
\left(\begin{array}{ll}
0 & 1 \\
0 & 0
\end{array}\right) \text {. }
$$

Here, $W_{n}(T)$ turns out to be all $n \times n$ matrices whose (ordinary) numerical radius is at most $\frac{1}{2}$.

We will say an operator $S \in L(\mathfrak{Q})$ is a projection of an operator $T \in L(\Re)$, if there is a subspace $\mathfrak{M}$ of $\Re$ such that $S$ is unitarily equivlent to $\left.P_{\mathfrak{M}} T\right|_{\mathfrak{M}}$. Note that we do not require $\mathfrak{M}$ to be invariant (or even semiinvariant) under $T$, so that projections of $T$ in general bear little resemblance to $T$. The following result gives a number of descriptions of the partial ordering of operators defined by the relation: $W_{n}(S) \subseteq W_{n}(T)$, for every $n \geqq 1$.

THEOREM 2. Let $S$ and $T$ be Hilbert space operators (acting, perhaps, on different spaces). Then the following are equivalent.

(i) $W_{n}(S) \subseteq W_{n}(T), n \geqq 1$.

(ii) $\|A \otimes I+B \otimes S\| \leqq\|A \otimes I+B \otimes T\|$, for every pair $A, B$ of $n \times n$ matrices, and every $n \geqq 1$.

(iii) Every finite-dimensional projection of $S$ is a projection of $\pi(T)$, for some *-representation $\pi$ of $C^{*}(T)$ (which may depend on the particular projection of $S$ ).

(iv) $S$ is a projection of $\pi(T)$, for some *-representation $\pi$ of $C^{*}(T)$.

(v) (For normal $S$ and $T$ ) $\operatorname{sp}(S)$ is contained in the convex hull of $\operatorname{sp}(T)$. 
(vi) (For $T$ compact and irreducible) $S$ is a projection of some multiple $I \otimes T$ of $T$.

3. Compact operators. The structure of $C^{*}$-subalgebras of $L C(\mathfrak{S})$ is well known, and leads to the conclusion that every compact operator is an (orthogonal) direct sum of irreducible compact operators. The same line of reasoning shows that the problem of classifying general pairs of compact operators (to unitary equivalence) reduces quickly to the case where both operators are irreducible. We now come to the main result.

THEOREM 3. Let $S$ and $T$ be irreducible compact operators on respective Hilbert spaces $\mathfrak{S}$ and $\Re$. If $\mathfrak{W}_{n}(S)=\mathfrak{W}_{n}(T)$ for every $n \geqq 1$, then $S$ and $T$ are unitarily equivalent.

If $\mathfrak{S}$ and $\Omega$ are of dimension at most $n<\infty$, and if $W_{n}(S)=W_{n}(T)$, then $S$ and $T$ are unitarily equivalent.

The proof is a straightforward application of Theorem 1 , Theorem 2(i) and (ii), and a general result (Theorem 2.1.2) from [1]. Note that the converse of both assertions is trivially true, and in particular $\left\{W_{1}(T), W_{2}(T), \cdots\right\}$ gives a complete set of unitary invariants for an irreducible compact operator $T$.

4. Some other applications. We conclude with two different applications of Theorem 1 and the results of [1]. Let $T$ be an operator on a Hilbert space $\mathfrak{E}$, and let $\mathfrak{M}$ be a semiinvariant subspace for $T$. In dilation theory, one wants to know (intrinsic) conditions on the projection $P_{\mathfrak{M}} T \mid \mathfrak{M}$ which force $\mathfrak{M}$ to reduce $T$ (i.e., $T \mathfrak{M} \subseteq \mathfrak{M}$ and $T^{*} \mathfrak{M}$ $\subseteq \mathfrak{M})$. The following result gives such an answer, for arbitrary subspaces, when $T$ is a multiple $I \otimes T_{0}$ of an irreducible compact operator $T_{0}$.

THEOREM 4. Suppose $T \in L(\mathfrak{W})$ is a multiple of an irreducible compact operator, and let $\mathfrak{M}$ be a subspace of $\mathfrak{S}$. Suppose $\left.P_{\mathfrak{M}} T\right|_{\mathfrak{M}}$ is compact irreducible, and $\mathfrak{W}_{n}\left(\left.P_{\mathfrak{M}} T\right|_{\mathfrak{M}}\right)=\mathfrak{W}_{n}(T)$, for every $n \geqq 1$. Then $\mathfrak{M}$ reduces $T$.

Our second application has to do with factorization. Consider the following question: given an algebra of operators $a \subseteq L(\mathfrak{S})$, which positive operators on $\mathfrak{S}$ can be factored in the form $T^{*} T$ with $T$ in $a$ ? Without going into the origins of this question (c.f. references [2] and [3]), we will describe a broad class of operator algebras which are at the worst extreme, in the sense that the sum of any (finite of infinite) sequence of factorable operators is almost never factorable. 
THEOREM 5. Let $a$ be a subalgebra of $L(\$)$ such that some irreducible set of compact operators commutes with a. Let $T, T_{1}, T_{2}, \cdots$ belong to a, with $T$ invertible, and suppose $\sum T_{n}{ }^{*} T_{n}=T^{*} T$. Then each $T_{n}$ is a scalar multiple of $T$.

\section{REFERENCES}

1. W. B. Arveson, Subalgebras of $\mathrm{C}^{*}$-algebras, Acta Math. (to appear).

2. -, Analyticity in operator algebras, Amer. J. Math. 89 (1967), 578-642. MR $36 \# 6946$.

3. H. Helson and D. Lowdenslager, Prediction theory and Fourier series in Several variables. II, Acta Math. 106 (1961), 175-213. MR 31 \#562.

University of California, Berkeley, California 94720 